hat sich ergeben, dass bei Injektionen unlöslicher Hg-Salze in genügend grosser Dosis ein relativ grosser Prozentsatz der Frühsyphilide der Haut, soweit dieselben allgemeine Exantheme darstellen, analoge Veränderungen zeigt, dass dies nach unserer Schätzung in $100 \%$ der Fall ist. Freilich tritt diese Veränderung, die wir als Reaktion bezeichnen wollen, in verschiedenen Graden auf, wobei die eingangs geschilderte klassische Reaktion nur in einem Theil der Fälle vorkommt.

Nachdem wir die Reaktion in mehr als 60 Fällen festzustellen und zu studiren in der Lage waren, können wir dieselbe in folgender Weise schildern. Sie tritt, wie gesagt, ein, wenn ein hinreichendes Quantum $\mathrm{Hg}$ auf einmal erstmalig zur Resorption gelangt. Daraus geht hervor, dass man sie nicht bei interner Verabreichung erwarten darf, wohl aber bei der Inunktionskur à $4,0 \mathrm{Hg}$-Salbe - und hier natürlich nicht nur an dem Orte der jeweiligen Einreibung - und bei der Injektion unlöslicher Salze à 0,1 des betreffenden Salzes. Von diesen letzteren erstrecken sich unsere Erfahrungen nur auf Hydrargyrum salicylicum und Calomel. In 15-24 Stunden ist gewöhnlich das Bild des Exanthems mehr oder weniger verändert. Zunächst nimmt die Zahl der Effloreszenzen zu. Roseolen, die vorher nicht constatirt werden konnten, traten deutlich zu Tage, ebenso konnte, wenn solche festgestellt waren, fast in jedem Falle eine sichere Zunahme der Zahl der Flecke wahrgenommen werden. Gleichzeitig findet fast immer ein stärkeres Hervorheben der Effloreszenzen über das Hautniveau statt, sodass dieselben mehr einer Urtica oder Erythempapel gleichen, also ein succulentes Aussehen gewinnell. Eventuell kommt ein Confluiren des Ausschlags, namentlich am Stamm, zu Stande, wobei wiederholt grössere guirlandenförmige Figuren resultirten. Dazu kommt noch als etwas gänzlich Fremdartiges die Farbe, die noch gestern schmutzig mattroth war und die heute frischroth ist. Die Papel wird ebenfalls insofern alterirt, als sie eine hellrothe Areola bekommt oder zur Urtica wird. Pusteln erhalten hochrothe Höfe. Innerhalb $36-48$ Stunden weicht der frische arterielle Farbenton dem alten color tristis. Die Effloreszenzen sind entweder ganz verschwunden, und zwar im allgemeinen ohne Schuppung und ohne Pigmentbildung - in einzelnen Fällen kounte nachträgliche Schuppung oder Pigmentbildung beobachtet werden, - oder es ist, wenn es sich um Papeln oder Pusteln handelt, der rothe Hof verloren gegangen, und die Effloreszenz ist etwas mehr abgeflacht und hat ihr altes Aussehen wieder erhalten. Bei der annulären Roseola, dem rezidivirenden makulösen Syphilid, sprang nach 24 Stunden in einem Falle der Ring an manchen Stellen unter tangentialer Verschmelzung mit dem Nachbarring plastisch hellroth hervor, das blasse Centrum völlig im Hautniveau zurücklassend. Natürlich reagirten auch die gemischten Exantheme und darunter auch solche mit rezidivirendem makulösen und papulösen oder pustulösen Syphilid.

Will man hiernach von einem Kennzeichen des Phänomens sprechen, so muss man als das Charakteristische der Reaktion das Uncharakteristischwerden der syphilitischen Exantheme als solcher bezeichnen. Unter erheblicher Zunahme der Zahl der Effloreszenzen wird ein im Ausbruch befindliches Exanthem ïber Nacht an Intensität und Extensität plötzlich gesteigert oder ein bereits

II. Aus der Hautkrankenstation des städtischen Krankenhauses in Frankfurt a. M.

\title{
Ueber eine bei Syphilitischen vorkommende Quecksilberreaktion.
}

Von Oberarzt Dr. Karl Herxheimer und Stabsarzt a. D. Dr. Krause.

Schon vor mehreren Jahren hatte der eine von uns auffällige Veränderungen syphilitischer Exantheme nach Hg-Zuführung gesehen. Vor mehr als Jahresfrist machten wir dann wieder eine solche Beobachtung an einem mit makulösem Syphilid behafteten Patienten, die unsere Aufmerksamkeit rege machte. Das Exanthem war nämlich nach erstmaliger Einklatschung mit 4,0 grauer Quecksilbersalbe nach etwa 24 Stunden derart verändert, dass weder Grösse, noch Gestalt, noch Farbe der Einzeleffloreszenz mehr in der ursprünglichen Weise vorhanden war. Dieselbe wal grösser, über die Haut erhaben geworden und hatte eine hochrothe Farbe angenommen, besass also jetzt eher die Eigenschaften einer Effloreszenz des Erythema exsudativum multiforme. Nach abermals 24 Stunden war der Ausschlag völlig geschwunden. Dieser auffallende Vorgang veranlasste uns, bei neuen Fällen systematisch auf analoge Vorkommnisse zu fahnden. Thatsächlich im Verblassen befindliches Exanthem blüht wieder auf, in beiden Fällen mit frischrother Farbe. Während der Dauer der Reaktion zeichnet sich das syphilitische Exanthem gegenüber dem Erythema exsudativum inultiforme durch Fernbleiben der subjektiven Symptome wie Jucken, Brennen, Hitzegefühl, sowie meist durch das Fehlen der eventuellen Schuppung aus. Keiner unserer Patienten neigte zur Urticaria, bei keinem konnte ein Reizphänomen oder Urticaria factitia festgestellt werden. Die Reaktion trat immer nur nach erstmaliger Verabreichung grösserer Hg-Dosen ein, niemals später, auch wenn z. B. auf eine Hg-salicylicum-Injektion eine solche von Calomel folgte.

Es ist wohl zu beachten, dass die bisherige Schilderung sich lediglich auf die allgemeinen Exantheme der Frühsyphilis bezieht. Lokalisirte Syphilide, Primäraffekte, Papeln an den Genitalien der Männer und Frauen, unter den Mammae, in den Achselhöhlen, zeigten keine oder keine deutliche Reaktion. Vielleicht deshalb, weil bei diesen länger bestehenden und ausgedehnteren Gebilden sich eine intensive Endarteritis gebildet hatte und das $\mathrm{Hg}$ nicht in genügend grosser Dosis in den Krankheitsheerd gelangen konnte. In zwei Fällen von tuberösem Syphilid ferner glaubten wir eine Reaktion wahrzunehmen, verinissten dieselbe aber in einem Falle von Hautgummen. Es wäre auch nicht auffallend, wenn die Reaktion ge- 
rade bei kutaner Spätlues nicht sichtbar wäre. Was endlich die Schleimhautplaques des Frühstadiums angeht, so ist es möglich, dass die Reaktion daselbst eintritt, indessen waren wir nicht in einem einzigen Falle in der Lage. dieselbe mit Sicherheit festzustellen, was auf der Schleimhaut besonders schwierig ist.

Die Reaktion war aber nicht nur eine lokale, sondern oft auch eine allgemeine. Es wurde in einem erheblichen Prozentsatz der Fälle eine Temperatursteigerung bis über $38^{\circ}$ beobachtet. Freilich wollen wir nicht behaupten, dass in allen Fällen die Temperatursteigerung nach Einführung grösserer $\mathrm{Hg}$-Dosen als eine reaktive zu betrachten sei. Wenn sie jedoch gleichzeitig mit anderen Erscheinungen der Reaktion eintritt und bald verschwindet, dürfte sie in unserem Sinne zu verwerthen sein. Ferner war in einem Falle mit Veränderung des Exanthems und Temperatursteigerung eine Anschwellung der Lymphdrüsen verbunden, die nach 24 Stunden wieder wesentlich zurückgegangen war, sowie Appetitlosigkeit und Schweissausbruch. Im allgemeinen blieben subjektive Symptome aus, in einem Falle jedoch, den der eine von uns mit Herrn Kohnstamm (Königstein i. T.) zusammen beobachtete, steigerten sich bei einem Syphilitiker nach Injektion von 0,1 Hydrargyrum salicylicum die intensiven Kopfschmerzen ins Ungemessene, ebenso im Beginn der später von Kohnstamm eingeleiteten Schmierkur mit grauer Salbe, um bei deren Fortzetzung gänzlich zu verschwinden. Von einer Patientin wurden Halsschmerzen nach der ersten Einspritzung geklagt, die ebenfalls nach 24 Stunden verschwunden waren. Auf diese und analoge Angaben muss in Zukunft wohl geachtet werden.

Es ist kein Zweifel, dass in allen Fällen von Reaktion die Involution der Exantheme beschleunigt wurde, und dies um so mehr, je intensiver sich die Reaktion gestaltete. Man kann es geradezu als Gesetz aufstellen, dass die Schnelligkeit der Heilung der Effloreszenzen in direktem Verhältniss zur Intensität der Reaktion steht.

Nun könnte man vielleicht den Einwand erheben, dass es sich bei der Veränderung der Exantheme, wie wir sie geschildert haben, nicht um eine Reaktion, sondern um eine Intoxikation handele, dass also eine $\mathrm{Hg}$-Toxidermie vorliege, die sich von einer anderen Intoxikation um so weniger unterscheide, als auch hier bis zu einem gewissen Grade Temperaturerhöhungen vorhanden seien. Es wären bei so ausgedehnten $\mathrm{Hg}$-Erythemen freilich höhere Temperatursteigerungen $\mathrm{zu}$ erwarten. Ausserdem würde die Erhöhung der Körpertemperatur gewiss nicht gegen die Auffassung einer Reaktion sprechen, da .wir sie auch bei anderen Reaktionen sehen, so z. B. bei der Tuberkulinreaktion. Ganz zweifellos aber handelt es sich nicht um ein Quecksilberexanthem im gewöhnlichen Sinne, da gar kein Grund vorhanden wäre, dass sich das Exanthem elektiv an die syphilitischen Flecke und Papeln anschlösse. Gerade die Quecksilbererytheme verschonen mit Vorliebe die luetischen Produkte, indem sie zwischen denselben und sich einen weissen Hof lassen. Dies z. B. in den Fällen von Callomon (Allgemeine medizinische Centralzeitung 1900, No. 88) und von E. Hoffmann (Berliner klinische Wochenschrift 1902, No. 39 und 40). Es fehlt ferner das diffuse Auftreten, die längere Dauer und die „starke lamellöse Epidermisabschuppung“ (Lesser) der Hg-Exantheme. Um ganz sicher zu sein, haben wir bei Patienten, welche anderweitige Hautkrankheiten hatten und wegen früherer Syphilis intermittirende Kuren durchmachten, die Wirkung des $\mathrm{Hg}$ auf die Haut beobachtet. Es handelte sich im ganzen um 15 Personen, welche Injektionen von Hydrargyrum salicylicum à 0,1 erhielten. Zwei davon hatten Impetigo contagiosa, drei Impetigo vulgaris, fünf Psoriasis, einer Lichen ruber planus und vier Dermatitiden aus verschiedenen Ursachen. In keinem Falle war eine Spur von Reaktion wahrzunehmen. Andererseits haben wir frühsyphilitische Exantheme mit anderen Stoffen behandelt, nämlich mit Jodkali intern und andere mit Einreibungen von Terpentinsalben nach Art derjenigen mit Unguentum Hydrargyri cinereum. Auch hier war das Resultat bezüglich der Reaktion durchaus negativ.

Aus dem Gesagten erhellt zur Genüge die Berechtigung, eine "Reaktion" anzunehmen. Diese wird durch eine Ueberempfindlichkeit der syphilitisch erkrankten Zelle gegenüber dem $\mathrm{Hg}$ hervorgerufen, d. h. dadurch, dass das $\mathrm{Hg}$ bei seiner Bindung in der syphilitisch erkrankten Zelle eine grössere Schädigung hervorruft. Wir glauben das $\mathrm{Hg}$ den Toxinen an die Seite stellen zu dürfen, die nach den Versuchen von Ransom, Wassermann und Takaki, Kempner, Blumenthal an die giftempfindliche Zelle ge- bunden werden. Der Vorgang hat bereits zahlreiche Analoga. Man kann dabei zwischen spezifischen und nicht spezifischen Stoffen unterscheiden. $\mathrm{Zu}$ den ersteren würde das Tuberkulin (R. Koch) zu rechnen sein, das nicht, wie Koch zuerst annahm, eine Coagulationsnekrose, sondern eine perituberkulöse Entzündung hervorruft, ferner das Malleïn (Sommer, Deutsche Zeitschrift für Thiermedizin $\mathrm{Bd}$. XX) und das Trichophytin (Plato, Archiv für Dermatologie, 60, I). Grösser ist die Zahl der nicht spezifischen (d. h. ohne bakterielle Thätigkeit hervorgebrachten) Stoffe, welche den Autoren zufolge Reaktionen hervorgerufen haben. Dazu gehören nach Matthes die Stofíwechselprodukte des Bacillus pyocyaneus und prodigiosus, die Deuteroalbumose und die Peptone. Die von dein Nukleïn behauptete analoge Einwirkung auf das Lupusgewebe konnte von $H$. Kossel nicht bestätigt werden, ebenso wurde übereinstimmend von fast allen Autoren die von Hans Hebra behauptete Einwirkung von Thiosinamin, einem Additionsprodukte von Senföl und Ammoniak, auf tuberkulöse Prozesse negirt. Für den Thierkörper hat dann noch Löwit eine ganze Reihe von Stoffen gefunden, welche eine allgemeine Reaktion hervorrufen. Hierher gehören Stoffe der regressiven Metamorphose des Eiweiss: Hemialbumin, Pepton, Pepsin, Harnsäure, Harnstoff, ferner Organ- und Blutegelextrakte sowie Curare. Eine spezielle Analogie mit der unserigen weist die Jodkalireaktion bei Aktinomykose insofern auf, als es sich auch um einen nicht spezifischen anorganischen Stoff handelt. Es liegt bei dem beschriebenen Vorgange zweifellos eine Heilwirkung vor, und in diesem Sinne würde vielleicht auch die Arsenwirkung bei Lichen ruber und Psoriasis aufzufassen sein, die bei letzterer Erkrankung bei genügend hoher Dosis oft Röthe, vermehrte Schuppung und Pigmentbildung im Gefolge hat.

Durch die von uns constatirte Hg-Reaktion frühsyphilitischer Exantheme wird die Zahl der nicht spezifischen Stoffe, die Reaktionen hervorbringen können, vermehrt. Freilich ist dieselbe schon gesehen, aber wohl kaum in ihrer ganzen Bedeutung erkannt worden. Jarisch (Wiener medizinische Wochenschrift 1895 , No. 17) schreibt darüber: „Ich meine die Beobachtung einer Art Reaktion, welche sich in den ersten Tagen der Quecksilbereinverleibung bei mit Roseola syphilitica behafteten Kranken durch eine Steigerung der topischen Krankheitserscheinungen verräth. Die einzelnen Flecke treten nach 2-5 Einreibungen oder Injektionen sehr häufig viel deutlicher in Erscheinung als zuvor und scheinen hierdurch auch an Zahl vermehrt." Ferner sagt Jadassohn (Venerische Krankheiten, Ebstein und Schwalbe, S. 580): „Speziell die erste Roseola wird nach der ersten Hg-Darreichung manchmal unter Fieber plötzlich ausgedehnter und intensiver geröthet - - das ist wie eine Reaktion analog der Tuberkulin. wirkung -, oder es stellt sich, besonders nach den ersten Injektionen, nur Temperatursteigerung ein." Man sieht, beide Autoren haben den Kreis der Reaktion viel zu eng gezogen. Das ist die einzige Ausbeute aus der syphilidologischen Litteratur, und das ist sehr zu verwundern, da gewiss viele Syphilidologen wenigstens die gelegentliche Beobachtung gemacht haben, dass ein syphilitischer Ausschlag durch die Hg-Behandlung "herauskam“. Allerdings ist es fraglich, ob die sogenannte urtikarielle Roseola der Autoren nicht identisch ist mit der von uns geschilderten, urtikariell auf $\mathrm{Hg}$-Einverleibung reagirenden. Es wäre in diesem Falle der Kausalnexus unerkannt geblieben, denn sowohl in der deutschen, als in der französischen und englischen Litteratur wird die Roseola urticata als Unterart der Roseola angesehen, nicht aber als selbständige, wenn auch flüchtige Complikation.

Wir wurden durch Exzision einer 24 Stunden nach einer Injektion von Hydrargyrum salicylicum à 0,1 auf der Höhe der Veränderung befindlichen Macula in den Stand gesetzt, den Prozess auch histologisch zu untersuchen. In der Peripherie der Affektion war ein Infiltrat entlang den Gefässen des Coriums zu sehen, im Centrum war das Infiltrat nicht nur um die Gefässe angeordnet, sondern diffuser, und es befand sich nicht allein, wie in der Peripherie, in der Pars papillaris und subpapillaris, sondern reichte in die Pars reticularis corii herunter, besonders die Gegenden um die Talgdrüsen und Haarbälge bevorzugend, während Muskeln und Nervenstämme normal schienen. Färbungen mit Hämatoxylineosin und v. Gieson erwiesen den lymphocytären Charakter der Infiltrate, die theilweise ganz reichlich von Mastzellen durchsetzt waren. An den Stellen dieser zelligen Einlagerungen fehlten, wie die Behandlung mit Alauncarmin und Weigert'scher Färbung lehrte, die sonst normalen elastischen Fasern. In die Augen fiel 
ferner das um die Infiltrate am stärksten vorhandene Oedem des Bindegewebes, während die Epidermis davon frei blieb, besonders bei Tinktionen mit v. Gies on, Hämatoxylin-Eisenchlorid, polychromem Methylenblau.

Die geschilderte Reaktion syphilitischer Exantheme ist auch von nicht zu unterschätzender praktischer Bedeutung. Es ist gar keine Frage, dass häufig in der Praxis makulöse Exantheme vorkommen, die nicht diagnostizirt werden können, weil ihre Farbe nicht charakteristisch ist oder weil ihre Zahl so gering ist, dass man sie bei Abwesenheit anderer luetischer Symptome nicht diagnostiziren kann. Dasselbe gilt von gewissen papulösen Syphiliden die sich manchmal von am Stamm lokalisirten Follikulitiden kaum unterscheiden lassen. Da man in allen Fällen allgemeiner Exantheme nach unseren Untersuchungen im Stande ist, durch die Reaktion die Diagnose zu sichern, so bietet diese einen schätzenswerthen diagnostischen Behelf. Leider fehlt dieser $\mathrm{da}$, wo er noch nöthiger gewesen wäre, beim Primäraffekt, so dass für eine Reihe von Fällen dieser Art immer noch das Erscheinen sogenannter sekundärer Symptome abgewartet werden muss. 